\title{
Micro- and Nanosensors for Medical and Biological Measurement
}

\author{
Peter Rolfe ${ }^{*}, 1,2$ \\ ${ }^{1}$ Oxford BioHorizons Ltd., 12 Park View Rd., Berkhamsted, Hertfordshire, HP4 3EY, UK \\ ${ }^{2}$ Harbin Institute of Technology, Harbin, China
}

(Received October 11, 2011; accepted December 8, 2011)

Key words: biomedical measurement, micro/nanosensors, MEMS/NEMS, lab-on-a-chip, photonics, invasive and noninvasive sensors, biocompatibility, biomedical metrology

Micro- and nanosensors have evolved rapidly in the last few decades and they have expanding roles within biology and medicine, where measurement science and technology is of key importance. The targets for measurement include a huge number of simple and complex molecules, physical quantities such as pressure, force, displacement and flow, and electrical and magnetic phenomena arising from the heart, brain, muscles and nerves. Routine clinical care of patients currently benefits from the use of macro- and microscale sensors based on electrical, electrochemical, acoustic, piezoelectric and optical principles. Disposable electrodes for recording biopotentials, such as the electrocardiogram and electroencephalogram, are common, whereas invasive electrochemical and optical fibre sensors for pressure, blood gases and $\mathrm{pH}$ are useful in intensive care. Microscale immobilised enzyme glucose sensors are largely confined to the analysis of small blood samples, their invasive use still facing technical challenges. Sensors constructed to the nanoscale using quantum dots and carbon nanotubes are now rapidly emerging, being aimed at more complex biomolecules such as DNA. Nanoparticles in general and surface-enhanced Raman spectroscopy also play important roles in these developments. The impact of micro- and nanosensors on the fundamental understanding of major biomedical challenges and on clinical diagnosis and care are highlighted here.

\section{Introduction}

Sensors and instrumentation for medical and biological measurement evolved steadily in the first half of the 20th century, establishing certain diagnostic devices, such as the

${ }^{*}$ Corresponding author: e-mail: PeterRolfe@aol.com 
electrocardiograph, the electronic sphygmomanometer for blood pressure measurement, and physiological monitors for anaesthesia and intensive care, as the classical symbols of biomedical instrumentation within healthcare.(1) Although complete measurement systems comprising of sensors and the associated electronics and displays are, of course, essential, the very significant challenges of sensor design and construction have, over many decades, attracted particular attention. ${ }^{(2)}$ In recent years, therefore, there has been the emergence of increasingly sophisticated and complex sensing devices and these are being tested or utilised in a growing number of biomedical situations for a diverse range of target measurands.

The application areas for medical and biological sensors have been evolving as the nature and priorities of basic research and routine clinical care have themselves moved forward. At one end of the spectrum is the fundamental biological research that is needed, for example, to understand the molecular phenomena that are so important in controlling the behaviour of cells, tissues and organs, and this is now heavily dependent upon the use of sensors. Then, there are needs for sensors in physiologically based clinical research, acute clinical care, and emerging fields such as tissue engineering, regenerative medicine, nanomedicine, and drug discovery. At the other end of the spectrum are the growing needs to develop measurement systems to be used for selfdiagnosis and self-care by individuals in their home and during normal daily life. In the latter case, developments in wireless sensor networks are now aimed at achieving socalled ubiquitous healthcare, ${ }^{(3)}$ and sensors constitute key components of such systems.

The variety of target measurands of interest and importance is now extremely diverse. Physical variables, such as pressure, force, flow, and displacement, are important for both basic research as well as for routine clinical care in fields such as biomechanics, orthopaedics, cardiorespiratory medicine, and intensive care. The intrinsic electrochemical nature of biological cells can be interrogated through measurement of electrical fields, charge, and magnetic flux. Such measurements are important for cardiac, musculo-skeletal, and cerebral monitoring. Then, at the molecular level, sensors are needed for gases, ions, proteins, DNA, bacteria, and viruses, as well as many other chemical compounds of importance for diagnosis of diseases such as diabetes, kidney and liver failure, and cancer.

The practical sensor devices developed to meet these diverse needs have been based on a number of important sensing principles. The earliest medical sensors for pressure or stress/strain utilising electrical principles embodied in resistive bridge strain gauges served the field well, ${ }^{(4)}$ and these still have applications. ${ }^{(5)}$ Electrochemical sensors, especially those based on amperometry ${ }^{(6)}$ and potentiometry, ${ }^{(7,8)}$ have been and continue to be key contributors in the vast range of chemical measurements currently undertaken. The measurement of bioelectrical phenomena also depends on the use of electrodes, ${ }^{(9)}$ commonly used in the electrocardiograph (ECG), electroencephalograph (EEG) and electromyograph (EMG). Of major importance at present are sensors based on optical principles, including absorbance, reflectance, fluorescence, and interferometry. The use of optical fibres to achieve such measurements was an important early development, ${ }^{(10)}$ whereas planar fabrication techniques have also provided many options for complex optical sensor configurations. $^{(11)}$ 
It is evident that current sensor advances owe much to key technological innovations that have had a particular impact on device fabrication, improving reliability and complexity at the microscale as well as opening the door to nanoscale devices. For example, semiconductor chip fabrication based on lithographic techniques continues to achieve ever-decreasing feature dimensions, now in the range of 10-20 nm, through efforts aimed at developing laser sources with adequate power at wavelengths in the extreme ultraviolet band around $13 \mathrm{~nm}$. Important contributions have also been made by advances in such fields as micro- and nano-electromechanical systems (MEMS/ NEMS), ${ }^{(12)}$ microfluidics, ${ }^{(13)}$ lab-on-a-chip ${ }^{(14)}$ and photonics-based micro- and nanodevices and systems using optical waveguides and plasmonics. ${ }^{(15)}$ The progressive evolution of sensor fabrication technologies has moved the medical sensor field quite distinctly from the macroscale, through the microdomain and now into the nanoworld. ${ }^{(16)}$ This enables the complexities of cellular and intracellular processes to be examined in detail at the fundamental level. In fact, there have also been significant benefits to research in sensor science and technology from the inspiration that derives from natural biological sensory systems, ${ }^{(17)}$ for example, from the senses of sight, hearing, taste, smell and touch. ${ }^{(18)}$ This has led to important activities in so-called biomimicry and biomimetics. ${ }^{(19)}$

In this review, the evolution of micro- and nanosensors used for biological and medical measurement is explored by considering sensing techniques, theoretical issues and practical challenges as well as applications. It is recognised that the use of a dimensional prefix, such as macro-, micro-, or nano-, can be interpreted in different ways, leading to some misinterpretations. For example, some use the prefix to refer to the dimensions of the complete packaged sensor device, whereas others use it to indicate the size of the sensing element alone. A complete sensor device may be anything from $100 \mu \mathrm{m}$ to $5 \mathrm{~mm}$ in diameter, but the sensing element could be just $20 \mathrm{~nm}$ in diameter. A comparison between the dimensions of the measurement site and of the sensor is therefore presented here. Also, the different interpretations of the dimensional prefix are highlighted in an effort to clarify the variants of sensor terminology currently being used by researchers, users, and manufacturers.

\section{Sensor Design Constraints}

\subsection{Measurands}

The recognised biomedical measurement application areas that exist have created demands for sensors in terms of both the required measurands and the constraints on sensor performance and physical configuration, including size and form. Table 1 shows a broad classification of the measurands and the biomedical systems and measurement sites appropriate for biomedical measurements.

The measurement of physical variables includes pressure, force, flow, displacement, and acceleration. Temperature measurement is also a basic requirement, especially for clinical monitoring. Among the chemical species of importance, oxygen, $\mathrm{O}_{2}$, and carbon dioxide, $\mathrm{CO}_{2}$, are vital in clinical care as well as in basic research. Measurement of nitric oxide, NO, has been of major interest since its roles in fundamental cellular processes became known. ${ }^{(20)}$ Sensors are needed for the measurement of a number of 
Table 1

Broad classification of measurands and measurement sites.

\begin{tabular}{lll}
\hline Type & \multicolumn{1}{c}{ Measurand } & \multicolumn{1}{c}{ Systems and sites } \\
\hline Physical & Pressure, force, flow, displacement, & Cardiac, respiratory, skeletal, gastro- \\
& acceleration, temperature & intestinal, central nervous system \\
Chemical & $\mathrm{O}_{2}, \mathrm{CO}_{2}, \mathrm{NO}, \mathrm{pH}, \mathrm{Ca}^{++}, \mathrm{K}^{+}, \mathrm{Na}^{+}$, & Intravascular, renal, hepatic, tissue, \\
& $\mathrm{Cl}^{-}, \mathrm{C}_{6} \mathrm{H}_{12} \mathrm{O}_{6}$ glucose, $\left(\mathrm{NH}_{2}\right)_{2} \mathrm{CO}$ urea, & noninvasive, breath, urine, saliva, in \\
& $\left(\mathrm{CH}_{3}\right)_{2} \mathrm{CO}$ acetone, bacteria, DNA, & vitro \\
& virus, biomarkers, drugs, antigen, & \\
& antibody & \\
Electrical, magnetic & ECG, EMG, EEG, MCG, MEG, EOG & Cardiac, cerebral, muscles, ocular \\
\hline
\end{tabular}

ions, particularly $\mathrm{H}^{+}, \mathrm{Ca}^{++}, \mathrm{K}^{+}, \mathrm{Na}^{+}$, and $\mathrm{Cl}^{-}$. These ions have profound influences on the behaviour of cells, which in turn control many physiological processes. Measurements of a variety of other more complex chemical compounds, such as glucose, which is of major importance in diabetes, urea and acetone, ${ }^{(21)}$ of relevance to kidney function, bring further significant challenges for sensor design and construction. Electrical signals including the so-called biopotentials, such as the ECG, EMG, and EEG, remain important for clinical diagnosis but are now increasingly being reassessed alongside magnetic measurements. Magnetocardiography, MCG, and magnetoencephalography, MEG, might offer advantages over the ECG and EEG.

\subsection{Measurement site}

Designing practical sensors that can be fabricated cost-effectively for reliable use, in either basic research or in routine clinical care, must take account of the intended site for the sensor to be located. This is important in order that the sensor geometry and overall dimensions can be designed to meet all practical and safety constraints. In addition to sensor shape, form and dimensions, it is also vital that the materials from which the devices are fabricated are chosen to ensure that any materials-related risks are minimised. In this context, the investigation of the biocompatibility of sensors is vital, ${ }^{(22,23)}$ and the materials from which the devices are fabricated are key factors in this.

There are three broad categories of measurement site that are helpful when considering these matters of sensor configuration and materials:

a) Invasive measurement. This involves the placement of the sensing device within living tissues, in either animal or human subjects. Devices may be inserted into an artery or vein, or be placed within the gastrointestinal tract or respiratory tract. Invasive sensors may also be inserted into cells or tissues being cultured in the laboratory.

b) Noninvasive measurement. ${ }^{(24)}$ In this case, the sensor is positioned on or near the surface of the living tissue. For clinical monitoring, this typically involves fixation of the sensing device to the skin surface. In cell culture bioreactors, it may involve sensors positioned either outside the culture chamber, or at the surface of a single cultured cell. 
c) Ex vivo and in vitro measurement. This involves the sensing device being situated within a sample chamber or other apparatus where it analyses the sample, which could be taken from a patient or experimental animal or it could be taken from a bioreactor.

With invasive and noninvasive measurement techniques, the sensor is required to capture data directly from the intact subject, patient, or organism. For this reason, both types of measurement are also referred to as being in vivo, literally "within the intact living organism". On the other hand, both ex vivo and in vitro mean "outside the intact living organism", but the latter specifically means "in glass". Thus, ex vivo and in vitro measurements particularly relate to the analysis of samples removed from the intact organism and such samples include blood, urine, saliva, breath, tissue or cells.

\subsection{Sensor device dimensions}

The physical size of the sensor devices used for the very wide range of work in biology and medicine is an important factor in terms of design and fabrication. Clearly, the size of the overall sensor device, its shape or form, must be appropriate for the measurement site and the sample volume in which the target measurands are located.

It is relevant to consider the dimensions of biological structures and human anatomical features for the scale of sensing devices to be put into context; this is shown in Table 2.

It can be seen that there are nanoscale items, structures, and features, not only as atoms and individual molecules but also as subcellular organelles, such as the cytoskeleton. DNA strands have subnanometre width dimensions but can be very long, as much as $3 \mathrm{~m}$ but are coiled into a ball in the nucleus. Whole cells, having a variety of shapes, vary from red blood cells that are discoid in shape and $2 \times 8 \mu \mathrm{m}^{2}$ in size, to

Table 2

This gives an approximate range of the sizes of elements, structures, features, tissues and organs. The size range extends from less than $1 \mathrm{~nm}$ for atoms to several tens of centimetres for human organs.

\begin{tabular}{lrl}
\hline Structure/Feature & \multicolumn{1}{c}{ Size } & \multicolumn{1}{c}{ Comment } \\
\hline Atoms and molecules & $0.2-10 \mathrm{~nm}$ & \\
Cytoskeleton (dia) & $5-25 \mathrm{~nm}$ & $\begin{array}{l}\text { Actin filaments (AF), intermediate filaments (IF), } \\
\text { microtubules }\end{array}$ \\
DNA (length) & $0.34 \mathrm{~nm} \times N_{\mathrm{bp}}$ & $N_{\text {bp }}$ number of base pairs; 3 m total length \\
Organelles & $1-5 \mu \mathrm{m}$ & Intracellular elements \\
Cell nucleus & $5 \mu \mathrm{m}$ & \\
Mitochondria & $0.1 \times 2 \mu \mathrm{m}$ & The cell's 'power house' \\
Cells & $5-100 \mu \mathrm{m}$ & Cell shapes are diverse \\
Red blood cells & $2 \times 8 \mu \mathrm{m}$ & Discoid in shape \\
Microvasculature (dia) & $5-10 \mu \mathrm{m}$ & Capillaries, venules \\
Arteries, veins & $1-200 \mathrm{~mm}$ & \\
Kidney, heart, brain & $10-20 \mathrm{~cm}$ & \\
\hline
\end{tabular}


skeletal muscle cells that can be $100 \mu \mathrm{m}$ in diameter and $3 \mathrm{~cm}$ long. Blood vessels are important in the context of sensor placement within the body since they can provide convenient access to blood, which is a key fluid for biochemical analysis as well as for direct blood pressure and flow measurement.

Measurements made ex vivo/in vitro frequently involve the removal of a sample from the subject or organism and placement within an analytical chamber. The nature of the sample and the available volume may impose limits on both the sensor and chamber in which the measurement is made. Examples of such samples are breath, blood, urine, faeces, saliva, and biopsy tissues, including both hard tissues such as bone and the more common soft tissues. Blood and other fluid sample volumes may be as large as $1-5 \mathrm{~mL}$ from human adults but as low as $50 \mu \mathrm{L}$ from premature babies and laboratory animals. In the widely used microarrays ${ }^{(25)}$ for protein and DNA analysis, a typical sample volume is $10 \mu \mathrm{L}$.

In situations where it is important to reduce the sample volume needed, or to minimise the influence that the mere presence of the sensor has on the biological environment in which it is situated, there have always been efforts to reduce the overall sensor device size. This is where advances in fabrication methods and technologies, such as MEMS and NEMS, play crucial roles and where recent developments have moved the state-of-the-art in terms of sensing phenomena firmly from the microdomain into the nanoworld.

The terminology of sensing devices based on dimensional criteria can be open to interpretation, and may, therefore, only be of limited value, unless there is agreement among users as to the specific meaning of dimensional prefixes. As has already been mentioned above, complete sensor devices usually require appropriate packaging and, in many cases, this significantly increases the overall dimensions. The notable exceptions to this are dye molecules and nanoparticles that can be delivered to invasive sites, where they sense the target measurand, yet may be interrogated, or read-out, remotely through externally applied EM or magnetic fields. In the case of these two examples, dyes and nanoparticles, there is no packaging required for the sensor and so it is valid to use the term nanosensor for the active element even if very large instrumentation is needed to complete the measurement system. Some nanoparticles, such as nanowires, can be used as the basis of sensor devices in which they are combined with other elements, such as transistors, in order to fabricate a complete device. In this case, the sensor is still a nanosensor but the complete device, for example, a virus sensor, is a large-scale device of several $\mathrm{mm}^{2}$. In this case, there is no real merit in using a dimensional prefix to describe the complete device, but it is likely to be branded a nanosensor simply because it incorporates such a sensor.

Further terminological issues arise in the field of electrodes used for electrical and electrochemical measurements. Some electrodes are used for intracellular measurement and they therefore need to have an active tip with a diameter of $1-10 \mu \mathrm{m}$. This sensing part is clearly a microsensor. However, the shank of the electrode is considerably larger, in the region of $1 \mathrm{~mm}$, to achieve the desired robustness for practical use. Thus, the device consists of a microscale sensing tip and a macroscale support. In this field, the complete devices are universally referred to as microelectrodes despite the fact that the 
overall device is macroscale. A similar situation arises with sensors used for direct intraarterial pressure measurement, where, for example, a 50- $\mu \mathrm{m}$-diameter fibre optic sensor is mounted on the tip of a catheter $3-7 \mathrm{~mm}$ in diameter. Strictly speaking, this overall device is not a microsensor, although it does contain one.

\section{Key Sensing Principles and Techniques}

Within the vast and growing fields of micro- and nanosensors, there are several key underlying sensing principles upon which practical sensors are based. Two major classes of sensing principles are, firstly, electrical and electrochemical sensing and, secondly, photonic or optical sensing. Devices based on these two classes of sensing have played such important roles in biomedical measurement as to warrant special description of the most important underlying principles.

\subsection{Electrical and electrochemical sensing}

Some of the most widely used biomedical sensors are based on electrical and electrochemical principles. These are convenient in that they usually combine the sensing element with the transducing function to produce a current or a potential in proportion to the target measurands.

\subsection{1 $\mathrm{Ag} / \mathrm{AgCl}$ reference electrode}

The simplest examples of electrochemical sensors are seen in the electrodes used for biopotential recording, as in ECG, EMG, EOG, and EEG. These electrodes are usually macroscale, merely being required to collect small electrical potentials $(0.1-1.0 \mathrm{mV})$ and feed them to a high-gain high-input-impedance amplifier. They can, nevertheless, be of microscale. ${ }^{(26)}$ They mostly utilise the all-important silver/silver chloride (Ag/ $\mathrm{AgCl})$ electrode, which, in the presence of a high concentration of chloride ions, $\mathrm{Cl}^{-}$, can establish a very stable half-cell potential. For this reason, the $\mathrm{Ag} / \mathrm{AgCl}$ electrode is used as a reference electrode in many other applications, including in a variety of microscale devices.

$\mathrm{Ag} / \mathrm{AgCl}$ electrodes are used with an electrolyte to supply the very necessary $\mathrm{Cl}^{-}$ions, and typically, this is $\mathrm{KCl}$. Then, metal $\mathrm{Ag}$ produces $\mathrm{Ag}^{+}$ions in the electrolyte solution and these combine reversibly with the $\mathrm{Cl}^{-}$ions provided by the electrolyte. A balance is achieved by electrons in the Ag metal flowing through a connecting wire. The two chemical relationships are as follows.

$$
\begin{gathered}
\mathrm{Ag}^{+}+\mathrm{Cl}^{-} \leftrightharpoons \mathrm{AgCl} \\
\mathrm{Ag} \leftrightharpoons \mathrm{Ag}^{+}+\mathrm{e}^{-}
\end{gathered}
$$

In addition to its use for collecting biopotentials, the $\mathrm{Ag} / \mathrm{AgCl}$ electrode is widely used in chemical sensors as a stable reference to allow a current or potential to be measured. The Nernst equation is used frequently in connection with electrode or biological cell potentials, and it can be written as 


$$
E=E^{0}-\frac{R T}{n F} \ln Q
$$

where $E$ is the cell potential under nonstandard conditions, $E^{0}$ is the standard cell potential, $R$ is the universal gas constant, $T$ is the absolute temperature, $n$ is the valency, $F$ is Faraday's constant, and $Q$ is the reaction quotient, which is the ratio of the concentration of products to the concentration of reactants. Substituting for $T, n$, and $F$, and converting to $\log _{10}$ gives the familiar form:

$$
E=E^{0}-\frac{0.059}{n} \log _{10} Q
$$

Under some conditions, the reaction quotient may equal unity and the cell potential will then simply be equal to the standard cell potential of $+0.223 \mathrm{~V}$.

\subsubsection{Amperometry}

Of particular interest among electrochemical approaches are amperometric and potentiometric sensors for gases, ions, and metabolites.

The simplest amperometric sensor, which is amenable to microscale fabrication, comprises of a noble metal working electrode ( $\mathrm{Au}, \mathrm{Ag}, \mathrm{Pt})$ held at an appropriate potential with respect to a $\mathrm{Ag} / \mathrm{AgCl}$ reference for reduction or oxidation of the target measurands. A current is then produced in proportion to the concentration of the measurands. There has been special interest in the measurement of $\mathrm{O}_{2}$ and $\mathrm{H}_{2} \mathrm{O}_{2}$ with such sensors.

The practical use of amperometric microsensors in biological fluids, such as blood, requires the use of permeable polymer membranes to surround the electrochemical cell so that diffusion of the target molecule to the working electrode can be controlled. With this arrangement, it is possible to achieve a useful relationship between current and concentration derived using Faraday's law and Fick's law:

$$
I_{\mathrm{d}}=n \frac{F A D_{\mathrm{s}}}{a} c_{\mathrm{s}}
$$

where $I_{\mathrm{d}}$ is the diffusion current, $n$ is valency, $F$ is Faraday's constant, $A$ is the area of the working electrode, $D_{\mathrm{s}}$ is the diffusion coefficient of the measurand in the membrane, $a$ is the membrane thickness, and $c_{\mathrm{s}}$ is the concentration of the measurand.

\subsubsection{Potentiometry}

The second major electrochemical method with which to create sensors is potentiometry. This method is particularly useful for the measurement of ions and it operates by generating a potential difference across an ion-selective membrane in proportion to ion activity. Ion-selective membranes have been mostly based on glasses, but polymer materials containing appropriate ionophores have been increasingly used. For the commonly used $\mathrm{pH}$ electrode, the relationship between the hydrogen ion activity, 
$\left[H^{+}\right]$, and the potential difference appearing across the glass membrane, $E$, is given by

$$
E=E^{0}+2.3 \frac{R T}{F} \log _{10}\left[H^{+}\right] .
$$

We define $p H$ as

$$
p H=-\log \left[H^{+}\right]
$$

At room temperature, $2.3 R T / F=0.0592 \mathrm{~V}$, thus, by substitution, we then have the linear relationship between $E$ and $p H$ as

$$
E=E^{0}-0.0592 p H
$$

$\mathrm{pH}$ sensors based on ion-selective membranes are therefore assessed in part by the slope of their $E$ vs $p H$ response, which, to be in accord with an ideal Nernstian response, should be $-0.0592 \mathrm{~V}$.

\subsection{Photonic sensing principles}

The many types of interaction between light, more broadly electromagnetic (EM) energy, and matter have provided extensive and diverse opportunities for the creation of sensing techniques and devices. In biomedical measurement, there is particular interest in those parts of the EM spectrum in the ultraviolet (UV), visible (Vis) and near infrared (NIR). This corresponds to wavelengths ranging from UV 220-400 $\mathrm{nm}$ (frequencies of $1,350-740 \mathrm{THz}$ ), Vis $380-760 \mathrm{~nm}$ (frequencies of 790-400 THz), and NIR 760-2,500 $\mathrm{nm}$ (frequencies of 400-120 THz). The photonic sensing methods in these EM bands might reasonably be considered to be inherently nanoscale and, in fact, reference to nanophotonics and nanooptics as specialist entities supports this idea. However, despite this, the fact remains that the complete optical devices or assemblies may be considerably larger.

\subsubsection{Absorption and scattering}

Much is known about the atomic and molecular interactions of EM waves or photons, and some of the most useful phenomena for sensing are absorption and scattering. As EM energy passes through matter, its intensity may diminish due to either absorptive or scattering process or a combination of both depending on the properties of the matter and the wavelength of the EM energy. In the case of purely absorptive matter, the LambertBeer law can be used to relate the incident intensity, $I_{\mathrm{o}}$, the concentration of the absorber, $[C]$, the transmitted intensity, $I$, the path length of light through the matter, $L$, and the molar extinction coefficient of the absorber, $\varepsilon$, as

$$
I=I_{\mathrm{o}} e^{-\varepsilon[C] L},
$$

from which the transmittance, $T$, and absorbance, $A$, can be derived: 


$$
\begin{gathered}
T=\log \left(I / I_{\mathrm{o}}\right)=-\varepsilon[C] L, \\
A=\log \left(I_{\mathrm{o}} / \mathrm{I}\right)=\varepsilon[C] L .
\end{gathered}
$$

The simplest use of these relationships for absorption measurement is in sensors where a dye is arranged to sense a chemical or physical event or process, such as oxygenation. For a more comprehensive spectrophotometric measurement, the wavelength of the interrogating EM energy is varied. Thus, for three absorbers and using three wavelengths (see Fig. 1):

$$
\begin{aligned}
& A_{\lambda 1}=\left(\varepsilon_{1, \lambda 1}[C]_{1}+\varepsilon_{2, \lambda 1}[C]_{2}+\varepsilon_{3, \lambda 1}[C]_{3}\right) L, \\
& A_{\lambda 2}=\left(\varepsilon_{1, \lambda 2}[C]_{1}+\varepsilon_{2, \lambda 2}[C]_{2}+\varepsilon_{3, \lambda 2}[C]_{3}\right) L, \\
& A_{\lambda 3}=\left(\varepsilon_{1, \lambda 3}[C]_{1}+\varepsilon_{2, \lambda 3}[C]_{2}+\varepsilon_{3, \lambda 3}[C]_{3}\right) L .
\end{aligned}
$$

These can be solved to allow calculation of $C_{1}, C_{2}$, and $C_{3}$.

The processes of elastic and inelastic scattering are also used extensively in biomedical sensing. One category of inelastic scattering is photoluminescence, which entails the absorption and reradiation of photons. One important type of photoluminescence is fluorescence in which the energy of the reemitted photons, although it occurs rapidly, within around $10 \mathrm{~ns}$, is less than that of the absorbed photons. This lower energy means that the wavelength of the emitted energy is increased as compared with that of the incident energy. This is given in the equation proposed by Planck:

$$
E=h c / \lambda=h v
$$

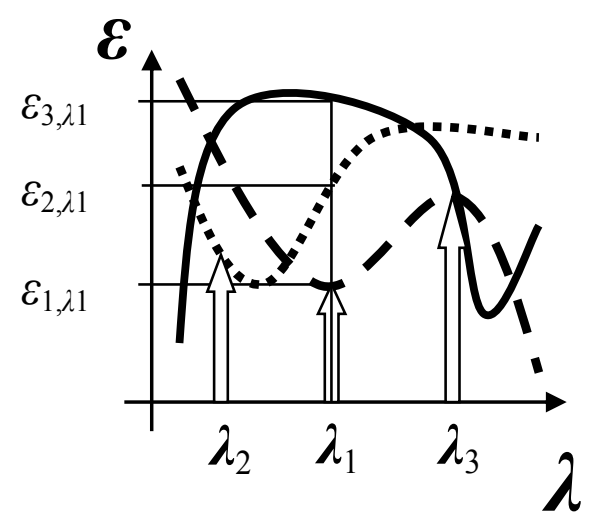

Fig. 1. This shows absorption spectra of three chemical species: dashed line, 1; dotted line, 2; solid line, 3. Interrogation with three wavelengths allows the three chemical concentrations to be calculated. Shown are the extinction coefficients of the three species at $\lambda_{1}$ (৫ Peter Rolfe). 
Here, $E$ is the energy, $h$ is Planck's constant, $c$ is the speed of light, $\lambda$ is the wavelength, and $v$ is frequency. Fluorescence is utilised in sensors in which a fluorophore sensitive to a target molecule is incorporated in some way with the sensing device. Typically, the fluorescence is quenched by the target molecule, $Q$, thereby creating an inverse relationship between fluorescence intensity and target concentration (see Fig. 2). This process is described using the Stern-Volmer Relationships:

$$
\left(I_{\mathrm{o}} / I-1\right)=\left(\tau_{\mathrm{o}} / \tau-1\right)=K_{\mathrm{sv}}[Q]=K_{\mathrm{q}} \tau_{\mathrm{o}}[Q] .
$$

Here, $I_{\mathrm{o}}$ and $I$ are the luminescence intensities in the absence and presence of the quenching analyte, and $\tau_{\mathrm{o}}$ and $\tau$ are the lifetimes of the excited state in the absence and presence of the quenching analyte, respectively. $K_{\mathrm{sv}}$ is the overall quenching constant and $K_{\mathrm{q}}$ is the bimolecular quenching constant. Another type of photoluminescence is phosphorescence in which the internal energy transfer involves intersystem crossing, which is a slow process compared with that taking place in fluorescence.

Another important type of inelastic scattering is that named after Raman. There are two forms: with Stokes Raman, the scattered photon has less energy than the absorbed photon; with anti-Stokes Raman, the emitted photon has more energy than the absorbed photon. Whereas fluorescence requires the incoming wave to have a specific frequency, the Raman effect can occur for any incoming frequency.

\subsubsection{Plasmonics}

Of growing importance in sensor development are approaches based on plasmonics. This field is concerned with the study and use of plasmons, which are quanta of plasma

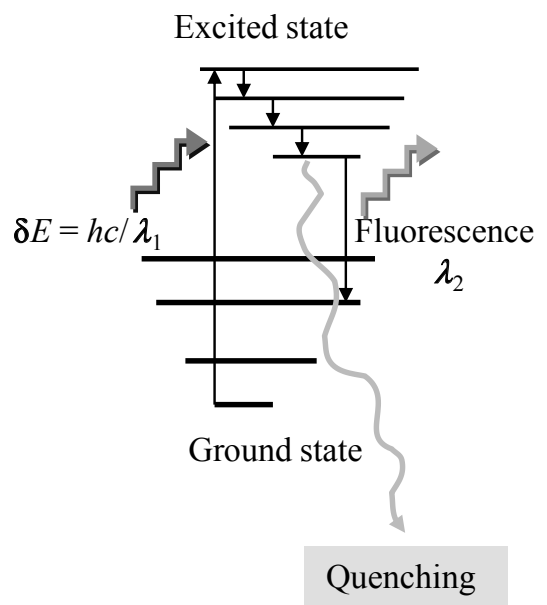

Fig. 2. This shows how fluorescence may be quenched, for example, by oxygen. 
oscillations and are considered as quasiparticles. Surface plasmons, which exist very close to surfaces, are particularly important since they interact with light, producing surface polaritons. These EM waves produced when light arrives at a dielectric-metal interface propagate parallel to the interface. The surface polaritons can be influenced by surface events such as particle attachment or change in refractive index, and this is used as the basis of sensing.

The excitation of surface plasmons by light, termed surface plasmon resonance (SPR), can be achieved by passing the light through a prism and creating an evanescent wave (EW) at the interface between the prism surface and air or a thin metal film (Fig. 3). The EW is created when the angle of incidence of the light beam at the interface is greater than the critical angle, producing total internal reflection (TIR). For different angles of the incident wave, the intensity of the reflected wave is at a minimum when resonance occurs. Changes of refractive index produced by surface events, such as antigenantibody binding, change the resonance angle and can therefore be quantified.

In order to calculate the depth of the 'field of view' of this kind of sensor, one examines the intensity of the $\mathrm{EW}, E_{\mathrm{EW}}$, as it diminishes exponentially in a direction normal to the interface:

$$
E_{\mathrm{EW}}=E_{\mathrm{o}} e^{-z / d_{2}}
$$

This then allows the depth of penetration, $d_{2}$, to be derived:

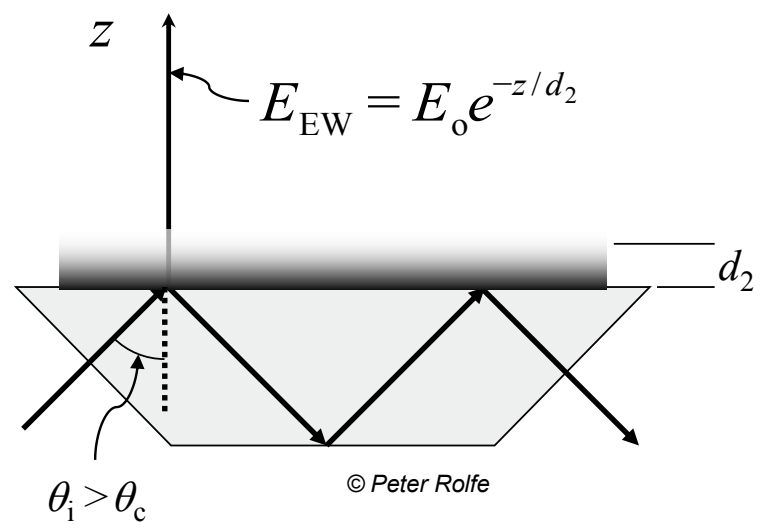

(a)

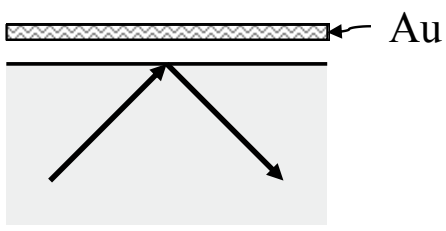

(b)

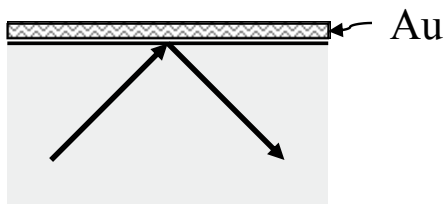

(c)

Fig. 3. (a) This shows the creation of an evanescent wave, $E_{\mathrm{EW}}$, at the interface between a prism and an external medium. Incident light approaches the interface at an angle, $\theta_{\mathrm{i}}$, which is greater than the critical angle $\theta_{\mathrm{c}}$. (b) This shows the addition of a thin Au film in the Otto configuration and (c) the Kretchmann configuration. 


$$
d_{2}=\lambda / 2 \pi n_{1} \sqrt{\left[\sin ^{2} \theta_{\mathrm{i}}-\left(n_{1} / n_{2}\right)^{2}\right]},
$$

where $n_{1}$ and $n_{2}$ are the refractive indices in the prism and external medium, respectively, and $\theta_{\mathrm{i}}$ is the angle of incidence and $\theta_{\mathrm{c}}$ is the critical angle. Deposition of a thin metallic film, of $\mathrm{Au}$ or $\mathrm{Ag}$, on or near the prism surface allows the EW to produce the surface polaritons. As shown in Fig. 3(b), in the Otto configuration, the metal film is positioned near the surface, whereas in the Kretchmann configuration (c), it is deposited directly on the surface. A further important variant of these plasmonic principles is to use nanoparticles with thin-film Au surfaces to produce localised surface plasmon resonance (LSPR). Moreover, it is useful to employ metallic films or particles to enhance Raman scatter, as in surface-enhanced Raman scatter (SERS).

\section{In Vivo Applications}

\subsection{Invasive measurement}

The insertion of measurement devices into a patient can be justified in several clinical situations. For example, patients who are under intensive care are likely already to have invasive procedures and the use of a sensor placed within tissues or the intravascular space could produce valuable information. In experimental animals, invasive devices may be justified in terms of the quality of the data that they could yield.

\subsubsection{General considerations}

The invasive approach to measurement involves the insertion of a device into the organism under investigation, be that a healthcare patient or a laboratory biological preparation, including an animal. This would appear to provide the most direct means with which to perform the measurement and may therefore be considered to constitute the preferred method when one considers matters such as accuracy and precision.

Metrological considerations are always important in biomedical measurement and these can become particularly challenging when an invasive approach is used. Sensors and their associated instrumentation need to be calibrated periodically, and there needs to be a quality control protocol in place to ensure that the derived data, especially when used for critical diagnosis or therapeutic monitoring, are of appropriate precision and accuracy.

\subsubsection{Tissue and intravascular sensors}

There are important clinical and basic research applications for invasive sensors that are designed to be inserted into the vascular system, or embedded in tissues, or inserted into single cells. The sensors of relevance in these areas can be either micro- or nanoscale, as will be seen from the dimensional data in Table 2. However, it is important to recognise that in many cases, the dimensions of the sensing element are often considerably smaller than the overall size of the device that is fabricated. Thus, a nanoscale sensor may well need to be much larger to be usable in practice. 
The established medical applications of invasive sensors are in the clinical care of critically ill patients who may be under intensive care, or coronary care or undergoing major surgery. The patients may be a fetus or newborn baby, children, or adults. The care of these patients involves the continuous monitoring of important physiological variables, such as blood gases, ions, metabolites, blood pressure, and blood flow. The aims are, firstly, to enable changes in the patient's condition to be detected as rapidly as possible and, secondly, to visualise and evaluate the effects of therapies so that these can be speedily optimised. Continuous real-time monitoring of many key variables can be achieved by placing sensors directly inside the vascular space. Figure 4 shows a microsensor at the tip of a flexible polymer catheter suitable for insertion into an artery or a vein where it can detect one or more target measurands.

The range of physical and chemical measurands for which intravascular sensors have been developed is extensive. Measurement of arterial and venous blood pressure is vital in critical care, and micropressure sensors based on semiconductor strain gauges are used routinely for this task. Such pressure measurement in these patients can be performed by inserting a flexible polymer catheter into an artery or vein in the wrist. In newborn babies, the cut umbilical cord provides access to both the arterial and venous spaces so that catheters can be inserted conveniently. Pressure measurement catheters are filled with saline and then an external pressure sensor can be attached to the catheter. Alternatively, a micropressure sensor can be inserted directly into the artery, as shown in Fig. 4. In this case, the sensing element is a semiconductor strain gauge but interferometric fibre optic sensors based on a Fabry-Perot cavity are also possible. ${ }^{(27)}$ Figure 5 shows such a device, in which the displacement of a flexible diaphragm is measured as a result of changes in the interference pattern between reflected and reference beams. Direct measurement of pressure inside the head (intracranial) or chest (intrapleural) may be required and these sensors are also suitable for this purpose.

The topic within the clinical microsensors field of most intense research effort has been that of chemical or molecular sensors. ${ }^{(28)}$ Devices have been developed for blood gases $\left(\mathrm{O}_{2}, \mathrm{CO}_{2}\right)$, ions $\left(\mathrm{H}^{+}, \mathrm{K}^{+}, \mathrm{Na}^{+}, \mathrm{Ca}^{++}, \mathrm{Cl}^{-}\right)$, molecules linked to metabolic processes (glucose, lactate, urea, creatinine), drugs, hormones, and microorganisms involved in

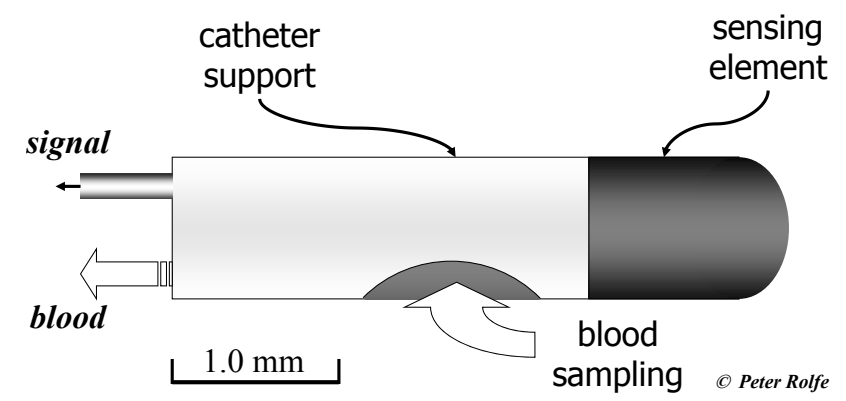

Fig. 4. This shows the general format of an intravascular sensor. 


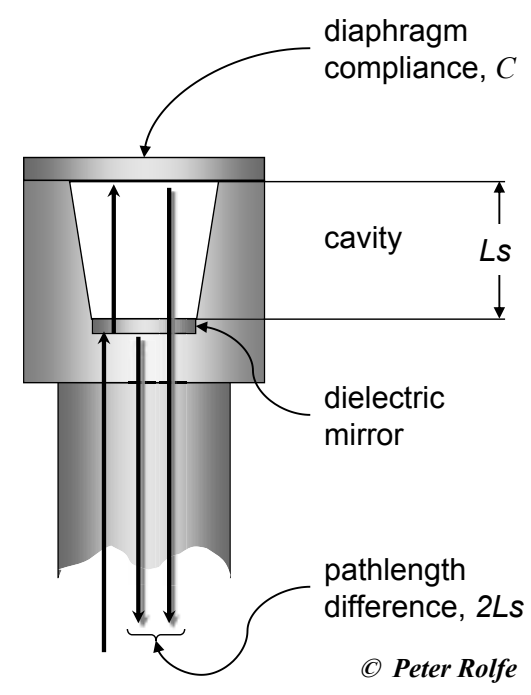

Fig. 5. This shows an optical pressure sensor based on a Fabry-Perot Cavity. The path length difference is measured by interferometry.

infection, such as bacteria and viruses. These sensors can be fabricated with microwires ( $\mathrm{Au}$, $\mathrm{Ag}$ and $\mathrm{Pt}$ ), of 1 to $20 \mu \mathrm{m}$ diameter, in amperometric and potentiometric electrochemical devices. Figure 6(a) shows a microsensor based on amperometry for insertion into tissue for measurement of $\mathrm{PO}_{2}$. This working electrode is held at $-600 \mathrm{mV}$ with respect to a reference electrode positioned adjacent to it.

Amperometric and potentiometric microsensors are also available for more complex molecules such as metabolites, drugs, and hormones. A glucose microsensor is shown in Fig. 6(b). Here, an enzyme is used to achieve the selective sensing function and so this is a true biosensor - a device with which sensing is achieved through biologically inspired means. This device has glucose oxidase enzyme immobilised within cellulose acetate, held between an outer glucose-permeable membrane of polyetherurethane and an inner layer of cellulose acetate. The enzyme catalyses the reaction between the substrate, glucose, and $\mathrm{O}_{2}$, thereby producing gluconic acid and $\mathrm{H}_{2} \mathrm{O}_{2}$. The reaction can be followed by measuring changes in $\mathrm{pH}$, or $\mathrm{PO}_{2}$ or the production of $\mathrm{H}_{2} \mathrm{O}_{2}$. The latter approach is shown in Fig. 6(b), with the oxidation of $\mathrm{H}_{2} \mathrm{O}_{2}$ being carried out at a $\mathrm{Au}$ anode. ${ }^{(29)}$

The measurement of ions (e.g., $\mathrm{pH}$ ) has been achieved with microscale glass electrodes, and these have been suitable for both tissue insertion as well as for incorporation into flexible catheters for intravascular use. In addition to the use of glass membranes for ion sensing, chemically sensitive field-effect transistors, CHEMFETs, can be used, not only for the measurement of ions but many other target molecules. For example, enzymes can once again be used to catalyse specific reactions that are then 


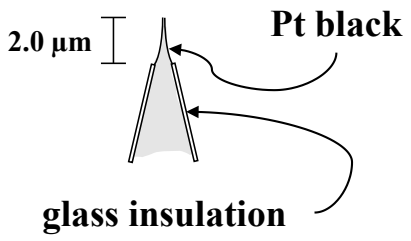

(a)

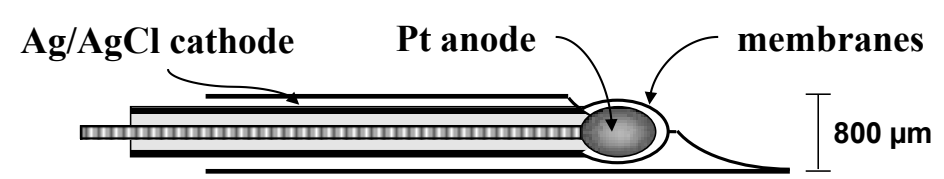

(b)

Ag/AgCl internal reference

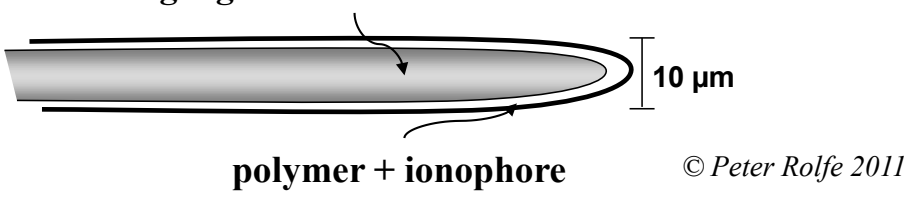

(c)

Fig. 6. Electrochemical sensors for invasive cell and tissue measurements. (a) A microneedle electrode for intracellular use. (b) An enzyme electrode using a triple-membrane structure contained within a hypodermic needle. (c) A coated wire ion-selective electrode using a polymer membrane containing an ionophore (see text).

followed by an ion-selective FET. A further alternative to glass ion-selective membranes is to use polymer membranes containing an ionophore for the target ion [see Fig 6(c)]. For example, the antibiotic valinomycin can be used as an ionophore for the $\mathrm{K}^{+}$ion. The advantage of the polymer ion-selective membrane is that they allow much simpler fabrication by dip-coating processes. There are many ionophores now available and others being developed to achieve greater selectivity and stability through the use of fluorous phases. ${ }^{(30)}$

Among the various types of invasive sensor, those designed for intravascular implantation are of major importance. Figure 7 shows an amperometric microsensor fabricated within a flexible tube that is suitable for the intravascular monitoring of $\mathrm{PO}_{2}$. A Pt working electrode and a $\mathrm{Ag} / \mathrm{AgCl}$ reference electrode together with a buffered $\mathrm{KCl}$ electrolyte are contained within an oxygen-permeable polymer chamber. As before, a current proportional to $\mathrm{PO}_{2}$ is produced by the reduction of the $\mathrm{O}_{2}$ diffusing into the chamber. This general approach has also been used for the fabrication of sensors to measure $\mathrm{PCO}_{2}, \mathrm{H}^{+}, \mathrm{K}^{+}$, glucose, urea, and $\mathrm{O}_{2}$ saturation. In the latter case, the sensor utilises spectrophotometric principles, based on the oxygen-dependant optical absorption spectra of oxy- and deoxyhaemoglobin. ${ }^{(31)}$

Optical sensing is a very important approach for clinical monitoring, and glass or polymer optical fibres are widely used as the basis of microsensor construction. These fibres are combined with chromophores or fluorophores to allow a wide range of 


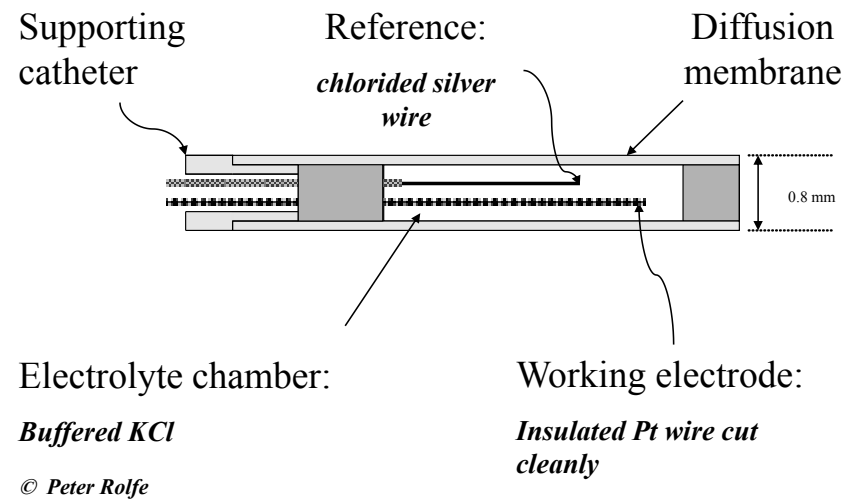

Fig. 7. An amperometric electrochemical sensor for invasive use.

species to be sensed, including gases and ions. ${ }^{(32)}$ Figure 8 shows a fibre optic sensor for $\mathrm{O}_{2}$ measurement. A fluorophore is adsorbed to microbeads within an $\mathrm{O}_{2}$-permeable envelope. Oxygen diffuses into the envelope and it quenches the fluorescence, which is detected by means of two optical fibres.

Despite the apparent advantages of invasive methods, the direct placement of an engineered device into a human subject or an animal preparation carries with it certain disadvantages and design challenges. In clinical situations, there are potential hazards to be considered, including infection, and this is also a significant concern in animal preparations. Furthermore, if devices are used within the subject or preparation for extended periods of time, such as a few days or longer, then undesirable interactions between the device and the biological environment can take place. Such interactions can produce immunological reactions from the host, as well as adversely affect the device performance. Interactions take place between the invasive sensor surfaces and the surrounding biological fluids; for example, proteins will be adsorbed to the sensor surface, cells can then adhere and this can lead to a coating of the sensor or encapsulation (see Fig. 9). Improving the biocompatibility of invasive sensors is still necessary and it is being approached with the use of materials that are derived from natural biomaterials, such as the cell membrane. ${ }^{(33,34)}$

\subsection{Noninvasive measurement}

The key feature of noninvasive measurement is that the whole process should be nondisturbing to the subject and to the target measurands. In the case of clinical monitoring, this means that not only is the patient as free as possible from any encumbrance of attached devices but also any potential hazards that might be associated with the measurement devices are minimised. When this is considered within the context of basic biological research, for example, in the study of cells that are cultured 

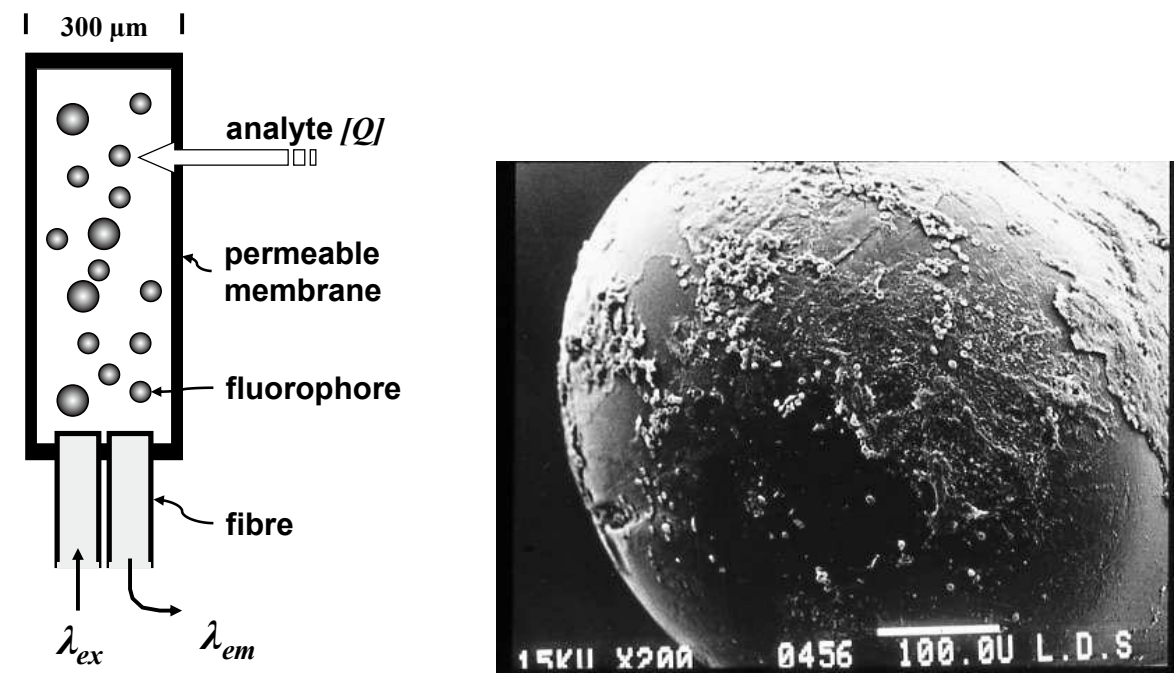

Fig. 8 (left). A photonic sensor based on fluorescence quenching. A fluorophore is adsorbed to microbeads. Two optical fibres carry exciting and emission beams (C Peter Rolfe).

Fig. 9 (right). This shows an SEM image of the surface of a membrane-covered intra-arterial sensor after removal from a patient. Proteins are seen adsorbed to the membrane surface and there are also cells attached ( Peter Rolfe).

in the laboratory, noninvasive measurement devices seek to avoid disruption of cellular integrity and behaviour.

\subsubsection{Skin surface chemical sensing}

An important sensor configuration for noninvasive measurement by attaching sensors to the patient on the surface of the body is shown in Fig. 10. The skin surface sensor is depicted, and the generalised structure of the skin, with three of its layers, is shown. Devices based on this approach are many and varied. Electroanalytical sensors can be used for the estimation of the arterial blood gases, $\mathrm{O}_{2}$ and $\mathrm{CO}_{2}$. Gases diffuse through skin and if the cutaneous blood flow is maximal, achieved by heat-induced vasodilatation at around $42^{\circ} \mathrm{C}$, then the skin surface $\mathrm{PO}_{2}$ and $\mathrm{PCO}_{2}$ are approximately equal to arterial values. ${ }^{(35-37)}$ With these electrically heated devices, $\mathrm{O}_{2}$ is measured with membranecovered Pt-cathode amperometric microsensors, whereas $\mathrm{CO}_{2}$ is derived from the $\mathrm{pH}$ of an electrolyte solution measured with a potentiometric $\mathrm{pH}$ sensor.

The indirect estimation of blood gas levels using skin surface devices requires validation, and this is done by comparison with data from the in vitro analysis of withdrawn blood samples. ${ }^{(38)}$ One limitation is in subjects who are in shock and whose peripheral circulation may be compromised. 


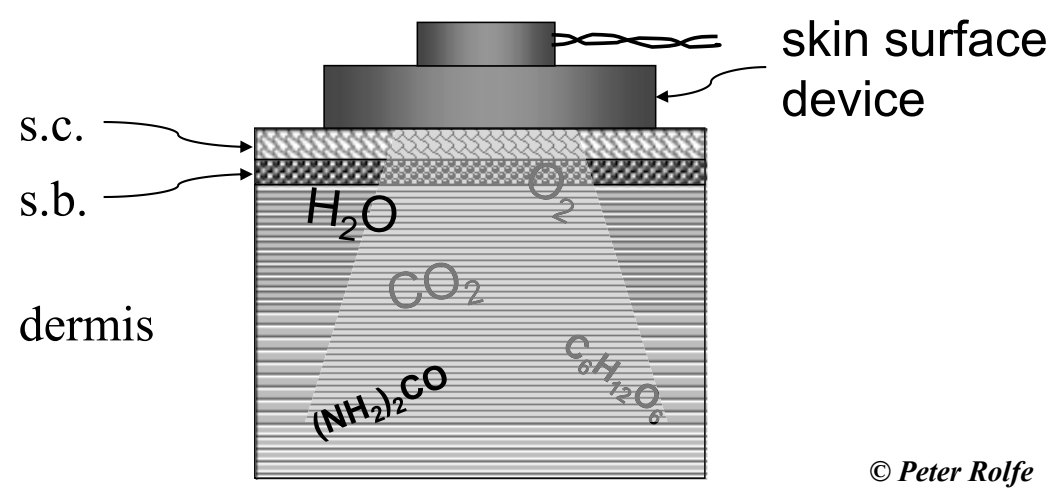

Fig. 10. Noninvasive measurement of tissue chemical constituents can be achieved with skin surface devices based on electrochemistry or photonics.

\subsubsection{Expired gas analysis}

For assessing breathing in some patients, it is useful to measure respired gas flow, volume and composition using micro-anemometers, pneumotachographs, and gas analysers. The analysis of expired gases has, until quite recently, only focussed on the measurement of $\mathrm{O}_{2}$ and $\mathrm{CO}_{2}$ for clinical purposes and, to a lesser extent for special respiratory studies, on $\mathrm{N}_{2}$ and helium. However, the detection of very low concentrations of expired molecules is of growing interest as a possible noninvasive way to detect important disease processes within the body. Early work in this area was carried out with large laboratory instruments, including mass spectrometers, but there are now possibilities to consider the use of microscale and indeed nanoscale sensor arrays. Such approaches are bioinspired, in that they attempt to mimic biological olfactory principles, embodied in the artificial nose and the e-nose. ${ }^{(39)}$ Fundamental cellular processes could be studied by this approach, and also, diseases such as kidney failure and cancer may possibly be detected early.

\subsubsection{Photonic tissue interrogation}

Near-infrared spectroscopy (NIRS) has been of interest for several decades as a possible means with which to measure blood oxygenation in deep tissues such as the brain and skeletal muscle. ${ }^{(40,41)}$ Biological tissues are relatively transparent to EM radiation in the NIR part of the spectrum, that is, for $\lambda=750-2,500 \mathrm{~nm}$. At the same time, there are useful absorption bands in this region for biological compounds that are important for both basic biological investigations and clinical diagnosis. For example, oxy- and deoxyhaemoglobin have well-known absorption spectra in the NIR, thereby allowing blood oxygenation and blood volume determinations in the brain and, in some circumstances, in other organs. Moreover, the intracellular respiratory enzyme 
cytochrome $\mathrm{aa}_{3}$ also has a characteristic absorption band at around $830 \mathrm{~nm}$, offering the possibility for intracellular oxidative processes to be studied.

In order to perform in vivo NIRS measurements, it is not necessary to use complex sensors. Instrumentation utilising laser diodes and semiconductor detectors are used, but the probes attached to the subject are quite simple, usually comprising of fibre optic bundles. The related technique of pulse oximetry, however, does use sensors with which to illuminate tissue and detect the diffusely backscattered or transmitted light. ${ }^{(42)}$ The absorption characteristics of oxy- and deoxyhaemoglobin are then used to calculate oxygen saturation. An important related technique is pulse glucometry for blood glucose estimation utilising the optical pulsatile signal from a finger. ${ }^{(43)}$

\section{Ex Vivo/In Vitro Measurement}

In addition to the importance of microsensors for continuous real-time clinical monitoring, there is growing activity in the development of multiple sensors, both micro and nano, for multianalyte processing of biological samples, such as blood, urine, saliva, breath, tissues, and cells. One important area of work aims to create the so-called 'labon-a-chip',(14) in many forms, whereby analyses previously carried out with large-scale laboratory instruments may be performed with simple handheld instruments. Once samples are removed from a patient, or from a laboratory preparation, a sample chamber is then used for the analysis and this can be a microfluidic or nanofluidic assembly incorporating sensing features. The rapid growth in the development of MEMS and, more recently, NEMS techniques has facilitated the creation of compact analytical platforms with large-scale sensing capabilities.

Analysis of blood samples can be achieved very near the patient by means of a sensor chip having an appropriate sample input (see Fig. 11). Once infused or aspirated into the device, the sample fluid enters a channel that is configured so as to bring it into contact with a number of sensors, either sequentially or simultaneously. The simplest device can

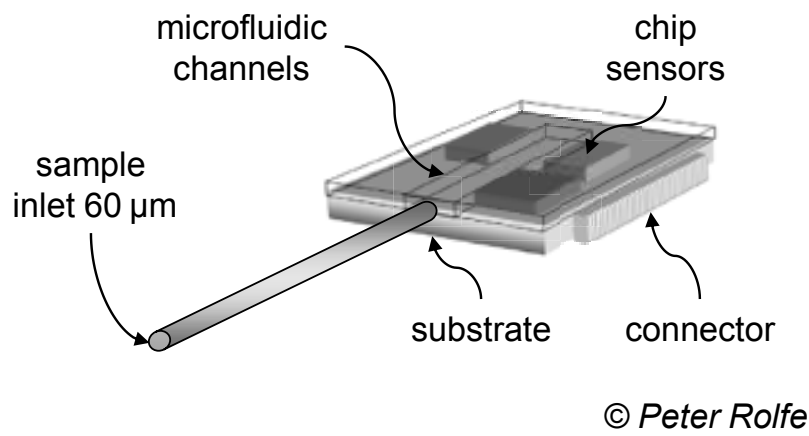

Fig. 11. Blood samples are taken from a microneedle and aspirated into a microfluidic chamber. Chip sensors for a variety of biological compounds then perform the analysis. 
use thick-film sensors, for example, for the measurement of blood gases $\left(\mathrm{PO}_{2}, \mathrm{PCO}_{2}\right)$ and $\mathrm{pH}$. Semiconductor sensors (ISFET, ChemFET, ImmunoFET) are now used in this type of device, offering a wide range of possible analytes including gases, ions, proteins, drugs, and hormones. Optical sensors based on semiconductor chips can also be included, offering the enormous power of spectrophotometric analysis. Surface plasmon resonance (SPR) is another powerful optical technique that has been utilised in sensors, for example, for receptor- or antigen-antibody-based sensors.

The application area where there has been the most significant impact of sensor arrays is that of genetic research, which has aimed to establish gene-related predisposition to disease. ${ }^{(25)}$ The DNA microarray (Fig. 12), or gene chip, consists of an array of specific segments of single-stranded DNA attached to, typically, a glass slide, and when the matching complementary segment of DNA combines with this (hybridisation), a fluorophore emits radiation. The pattern of fluorescent microdots is then analysed as an image. Microarrays are now being used to study the relationships between genes and the behaviour of cells, for example, to discover methods for the early detection of cancers, heart and cerebrovascular diseases, and in tissue engineering.

Copying nature, or biomimetics, provides a rich source of inspiration in sensor array development. ${ }^{(17,19)}$ Biological cells forming the basis of sensory systems for vision, hearing, olfaction (the e-nose $\left.{ }^{(39)}\right)$, touch, and taste have been emulated in solid-state

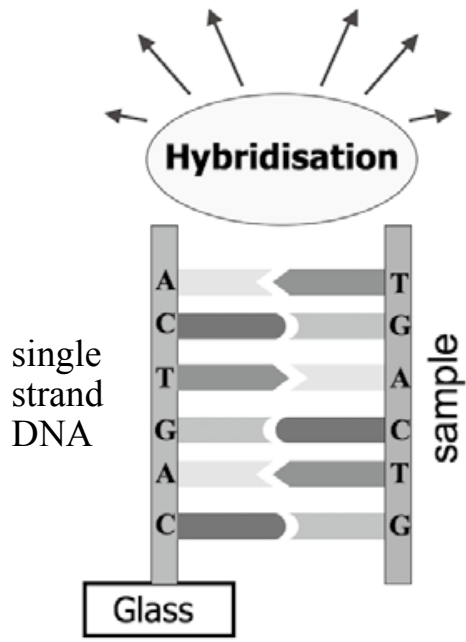

(a)

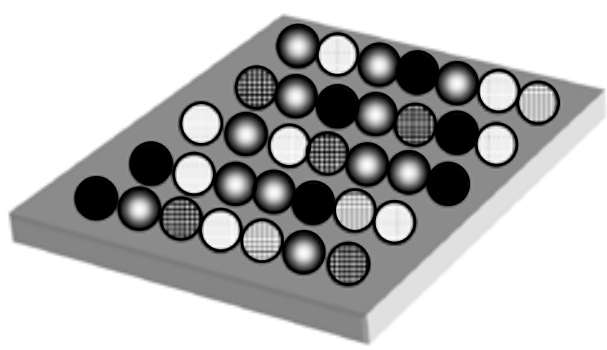

(b)

Fig. 12. This shows the principle of the DNA microarray. In (a), a single strand of DNA is attached to glass. When the complementary target is recognised, hybridisation occurs with fluorescence emission. In (b), an array of ssDNAs that have been hybridised displays the pattern of recognised targets. This is analysed by computer image processing (C Peter Rolfe). 
devices, producing biomimetic chips. These sensor arrays often combine processing in the form of neural networks to achieve very high selectivity, and they are beginning to have an impact on the new approach to organ repair and replacement within the field of cellular engineering. ${ }^{(17)}$

\section{Cell Research and Engineering}

\subsection{Towards nanoparticles}

Basic cell research continues to rely on advances in measurement technologies, including sensors and microscopy. The ability to culture cells in specialised bioreactors opens up numerous opportunities for micro- and nanosensor designs. Considering single cells, there are important constraints on sensor design firstly relating to scale and secondly to the issue of cell disruption (see $\S 2.3$ above). Although microneedle sensors, based on electrochemistry or photonics, have, over many years, been used for valuable work in single-cell experiments, their limitations in terms of disruption of cellular integrity and disturbance of normal behaviour are often questioned.

As with clinical measurement, described above in $\S 4$, measurement of tissue $\mathrm{PO}_{2}$ and $\mathrm{pH}$ has been of major interest for basic physiological systems research and, indeed, in single-cell studies. Microcirculation studies have been based on the use of $\mathrm{O}_{2}$ microelectrodes, of 1-10 $\mu \mathrm{m}$ diameter, as well as with the use of oxygen-quenched phosphorescent dyes. ${ }^{(44)}$ In fact, amperometric microsensors have been a key part of this field of research since the middle of the last century and devices have been designed for intracellular as well as skin surface measurement. ${ }^{(45)}$ The microscale Pt cathode, with or without a covering gas diffusion membrane, has been, once again, of significant value. More recent fabrication technologies have led to thin-film and thick-film devices with arrays of cathodes for spatial measurements.

Improvements of microneedle sensors continue to be reported. An ultramicro $\mathrm{Ag} / \mathrm{AgCl}$ reference electrode having a glass capillary tip outer diameter of $1.0 \mu \mathrm{m}$ and inner diameter of $0.5 \pm 0.2 \mu \mathrm{m}$ has been developed and its assessment for intracellular measurement reported. ${ }^{(26)}$ A salt bridge was used to make contact between an internal $300-\mu \mathrm{m}$-diameter chlorided silver wire rather than fabricating a Ag microtip.

Nanosensors are many and varied and are already having an impact on cell and tissue research. These sensors are based, among other things, on optical, electric, magnetic, acoustic, and immunological principles. ${ }^{(46)}$ Nanoparticles, NPs, such as nanoshells, nanowires, carbon nanotubes (CNT), and quantum dots (QDs), can be used to create such sensors. Gold nanoparticles (GNs) can sense their dielectric environment and this phenomenon can be harnessed in sensor design. The GNs can be functionalised with a molecular recognition element and thereby act as biosensors. Nanoshells, constructed with a spherical dielectric (e.g., silica) core surrounded by a gold or silver coating of a few nanometre thickness, can be interrogated using an EM wave at an appropriate wavelength, from UV to IR, to create a plasmon resonance. This significantly increases the light intensity, which is of benefit in the application of Raman spectroscopy, and these plasmon resonance effects are utilised in surface-enhanced Raman spectroscopy, SERS, and localised surface plasmon resonance, LSPR. 
Nanoparticle sensors have been developed for the measurement of a wide range of important measurands including $\mathrm{O}_{2}$, a number of ions, free radicals, and electric and magnetic fields. To measure $\mathrm{O}_{2}$, the NP can utilise the well-proven approach based on fluorescence quenching, by incorporating the standard ruthenium-based indicator dye, $\left[\mathrm{Ru}(\mathrm{dpp})_{3}\right]^{2+}$, together with a reference dye into the appropriate NP, e.g., silica. However, it has been shown that the precise choice of dye and the method of fabrication are important. ${ }^{(47,48)}$ NP sensors for ions, such as $\mathrm{H}^{+}, \mathrm{K}^{+}$, and $\mathrm{Ca}^{++}$, can use hydrophilic particles such as polyacrylamide, once again loaded with an appropriate dye. A different approach has been described for $\mathrm{pH}$ measurement in which a silica shell is used to encapsulate a rhodamine- $\beta$-iso-thiocyanate $\mathrm{H}^{+}$-sensitive core. ${ }^{(49)}$ In this case, a wide operating range of $\mathrm{pH} 3-10$ was achieved. This is important as has recently been reported, and the use of two fluorophores was described to achieve an intracellular $\mathrm{pH}$ range of 3.2-7.0. ${ }^{(50)}$

Nanowire sensors can utilise the measurement of conductivity change that takes place when a macromolecule binds to the nanowire surface. Figure 13 shows how a nanowire can be used to create antigen-antibody nanosensors, in this case, for the detection of a microorganism. This approach has been reported for the design of a virus nanosensor. ${ }^{(51)}$

Semiconductor QDs can be used effectively to act as fluorescent labels, having advantages over conventional dyes. They are more stable than conventional dyes, have a higher fluorescent yield, and have good resistance to photobleaching. Typically, the QD is a CdSe core surrounded by a $\mathrm{ZnS}$ shell, with an overall diameter of $2-10 \mathrm{~nm}$ (see Fig. 14). The anticipated elevation of an electron stimulated by a UV photon is given as

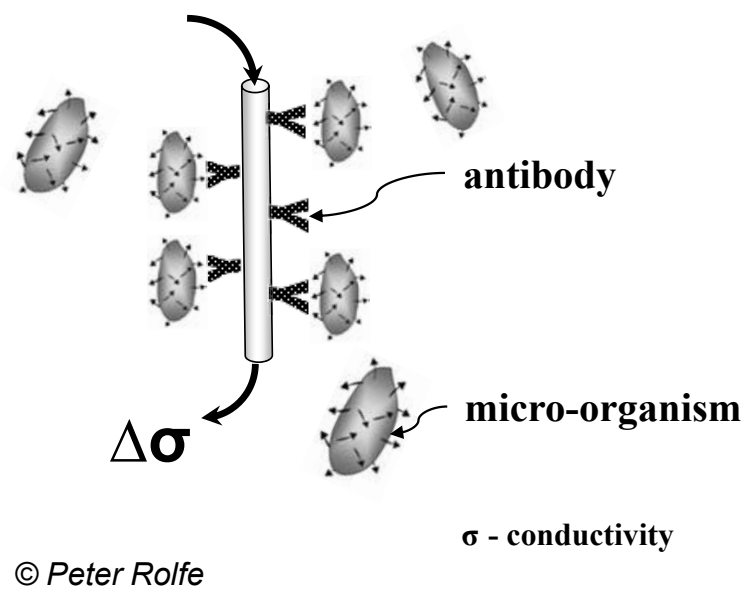

Fig. 13. This illustrates the use of a nanowire to create an antigen-antibody nanosensor for the detection of microorganisms. As antibodies recognise their target, the conductivity of the nanowire changes. 


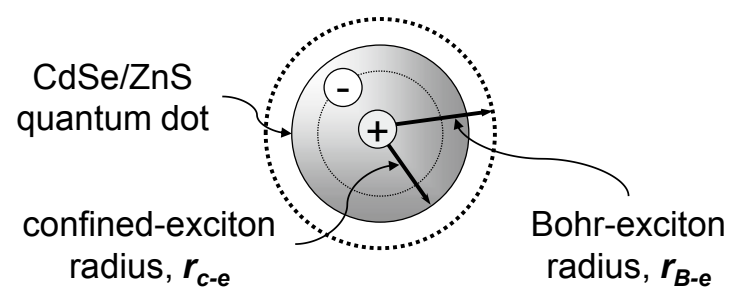

Fig. 14. This shows a quantum dot, QD, having a physical radius less than the Bohr-exciton radius for the $\mathrm{CdSe} / \mathrm{ZnS}$ material. The resulting confined-exciton radius leads to emission wavelengths that are tunable by choosing the QD size, ranging from 2-10 nm (C Peter Rolfe).

the Bohr-exciton radius, $r_{\mathrm{B}-\mathrm{e}}$, with an associated emission wavelength of $\lambda_{\mathrm{B}-\mathrm{e}}$ produced on return to ground state. However, the QD radius, $r_{\text {cee }}$, is chosen to be less than $r_{\mathrm{B}-\mathrm{e}}$, which leads to quantum confinement. Consequently, the confined emission wavelength, $\lambda_{\text {cee }}$, can be determined by choice of the QD radius. In cells and tissues, QDs can be used to localise or track mesenchymal stem cells when transplanted into tissues or when cocultured with other cell types. ${ }^{(52)}$ QDs are also now used in an increasing range of sensors, ${ }^{(53)}$ for example, based on fluorescence resonance energy transfer (FRET) for DNA analysis. ${ }^{(54)}$ Carbon nanotubes are also offering exciting possibilities for sensor fabrication, due to their unusual electrical and physical properties. A glucose biosensor has been reported, in which an array of CNTs have been attached to a platinum substrate, glucose oxidase has been immobilised to the CNTs, and direct electron transfer from the enzyme to the Pt electrode has been achieved. ${ }^{(55)}$

\subsection{Cells in bioreactors}

The fields of cellular engineering, tissue engineering, and tissue regeneration are evolving rapidly. The culture of cells and tissues allows experiments to be controlled, and the use of specialised bioreactors has become important for this. The evolution of bioreactors has also been accompanied by the development of comprehensive measurement techniques that allow improved observation as well as the control of the cell and tissue culture processes. ${ }^{(56)}$ Figure 15 shows a schematic of a bioreactor in which cells are stirred during the culture process. Three options for monitoring the processes are indicated: invasive, noninvasive, and shunt. This is very similar to clinical monitoring and, in fact, very similar sensors can be used in both applications. Invasive bioreactor monitoring is carried out with catheter-tip probes for $\mathrm{O}_{2}, \mathrm{CO}_{2}, \mathrm{pH}$, and glucose, whereas noninvasive monitoring can utilise NIR spectroscopy and fluorescencebased reporter films attached to the internal glass wall or window. Nanoparticle sensors are also suitable for use in bioreactors to enable the study of complex cellular processes.

The shunt measurement approach involves the transfer of bioreactor contents, including cells if necessary, through a measurement chamber for analysis. This is a very 


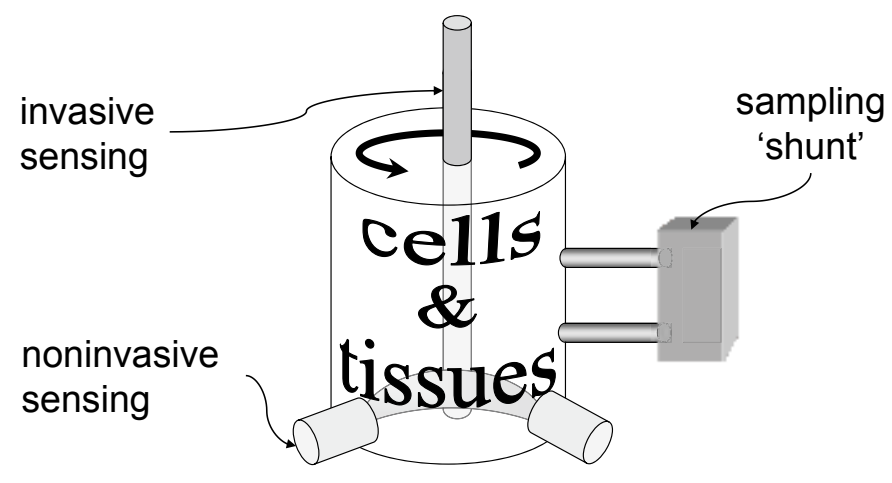

Fig. 15. This depicts a typical rotational bioreactor. Three approaches are possible for measurement: invasive sensing, noninvasive sensing, and sampling shunt. A variety of sensing techniques is possible including electroanalysis and a wide range of photonic methods, such as IR spectroscopy, fluorescence, and optical coherence tomography (C) Peter Rolfe).

versatile approach, allowing complex measurements to be made on a periodic basis. The measurement chamber may use individual sensors or a sensor array, based on the full range of measurement principles. Once again, NIR spectroscopy has much to offer, for example, to carry out protein analysis of the withdrawn samples. ${ }^{(57)}$ Vibrational spectra of proteins are also very important for analysis in the NIR region. The repeating backbone of proteins is responsible for the particular modes: $\mathrm{NH}$ stretch at $3,300 \mathrm{~cm}^{-1}$, CO stretch at 1,600 to $1,700 \mathrm{~cm}^{-1}$, and $\mathrm{NH}$ deformation at 1,500 to $1,550 \mathrm{~cm}^{-1}$.

An example of a specialised flow-through bioreactor is shown in Fig. 16. This assembly allows the monolayer culture of endothelial cells, with flow of nutrients and metabolic products. Individual sensors and sensor arrays can be included in this bioreactor depending on the experimental objectives. For example, metabolic processes can be followed using electrochemical and photonic sensors.

\section{Discussion and Conclusions}

There is enormous diversity in the range of micro- and nanosensors that have emerged over the last half-century or are now in the course of development. The focus on the scale of sensors becomes somewhat blurred when one fully takes into account the totality of a sensing device, but there is no doubt that the move into the nanoscale is of substantial importance and not just a technological oddity. Whilst the roles of macro- and microsensors continue to be vital in clinical care, for example to measure blood pressure, the ECG, and blood gases, the potential of the emerging families of nanosensors to make enormous contributions to basic research is evident. Nanoparticles (shells, spheres, rods, wires, tubes, and dots) are already being used in biological systems, including cells 


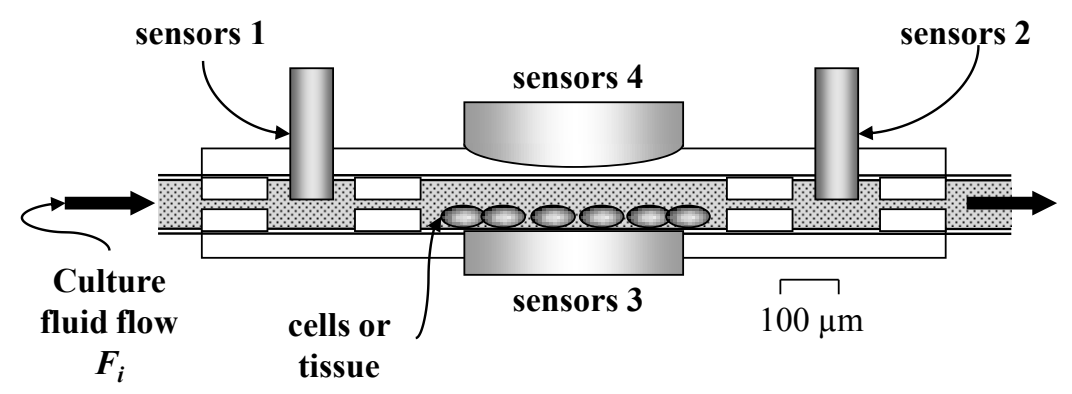

Fig. 16. This flow-through bioreactor allows basic studies on cell behaviour, from metabolic processes to cell signalling. The four sensor assemblies can be chosen according to the application and include electrochemical and photonic devices (C Peter Rolfe 2005).

and tissues, and also the lungs and gastrointestinal tract. The potential risks of NPs are recognised and are the subject of debate and research. For example, CdS quantum dots can liberate toxic cadmium if exposed to appropriate levels of UV. Clearly, therefore, every application must be examined thoroughly to evaluate risks.

Nanoscience could also contribute to ongoing developments of microsensors, for example, to help solve the problems associated with the biocompatibility of invasive sensors. It is known that interactions between implanted devices and biological fluids depend on surface properties and these could be tailored with nanomaterial strategies.

The micro/nanoworld offers exciting new prospects to achieve sensing of complex molecules. Research must continue so that the potential for these technologies to move us closer to achieving the early detection of serious illness through personal health screening in the home as well as in the hospital ultimately becomes a reality.

\section{Acknowledgements}

The author is grateful to students and staff who have worked with him at HIT, Harbin, China, the University of Genova, Italy, and Kanazawa University, Japan.

\section{References}

1 L. A. Geddes and L. E. Baker: Principles of Applied Biomedical Instrumentation (Wiley, New York, 1989).

2 P. A. Öberg, T. Togawa and F. A. Spelman: Sensors in Medicine and Health Care (Wiley-VCH, Weinheim, 2004).

3 R. S. Tolentino and S. Park: Int. J. Adv. Sci. Technol. 18 (2010) 1.

4 R. S. C. Cobbold: Transducers for Biomedical Instruments: Principles and Applications (Wiley, New York, 1974). 
5 W. G. Assunção, V. A. Barão, L. F. Tabata, E. A. Gomes, J. A. Delben and P. H. dos Santos: J. Craniofac. Surg. 20 (2009) 1173.

6 L. A. Geddes: Electrodes and the Measurement of Bioelectric Events (Wiley, New York, 1972).

7 W. Cha, Y.-C. Tung, M. E. Meyerhoff and S. Takayama: Anal. Chem. 82 (2010) 3300.

8 M. J. Martin and P. Rolfe: Anal. Proc. 23 (1986) 303.

9 A. Bratov, N. Abramova and A. Ipatov: Anal. Chim. Acta 678 (2010) 149.

10 J. I. Peterson, S. R. Goldstein, R. V. Fitzgerald and D. K. Buckhold: Anal. Chem. 52 (1980) 864.

11 S. M. Borisov, R. Seifner and I. Klimant: Anal. Bioanal. Chem. 400 (2011) 2463.

12 C. T. Leondes (ed.): MEMS/NEMS Handbook Techniques and Applications (Springer, New York, 2006).

13 B. Lin: Microfluidics: Technologies and Applications. Topics in Current Chemistry 304 (Springer, New York, 2011).

14 H. Craighead: Nature 442 (2006) 387.

15 P. Rolfe: Optoelectron. Instrum. Data Proc. 46 (2010) 324.

16 D. Son, S. Y. Park, B. Kim, J. T. Koh, T. H. Ki, S. An, D. Jang, G. T. Kim, W. Jhe and S. Hong: ACS Nano 5 (2011) 3888.

17 P. Rolfe: Eng. Sci. Educ. J. 6 (1997) 155.

18 J. Courville, J. Walsh and J. P. Cordeau: Science 138 (1962) 973.

19 R. Müller and R. Kuc: Bioinspiration Biomimetics 2 (2007) S146.

20 M. W. Radomski, R. M. Palmer and S. Moncada: Proc. Natl. Acad. Sci. U.S.A. 87 (1990) 5193.

21 C. Turner, C. Walton, S. Hoashi and M. Evans: J. Breath Res. 3 (2009) 046004.

22 N. Henninger, S. Woderer, H. M. Kloetzer, A. Staib, R. Gillen, L. Li, X. Yu, N. Gretz, B. Kraenzlin and J. Pill: Biosens. Bioelectron. 23 (2007) 26.

23 C. Yang, C. Zhao, L. Wold and K. R. Kaufman: Biosens. Bioelectron. 19 (2003) 51.

24 P. Rolfe: Non-Invasive Physiological Measurements, Vol. II (Academic Press, London, 1983).

25 M. Wiltgen and G. P. Tilz: Hematology 12 (2007) 271.

26 T. Kitade, K. Kitamura, S. Takegami, Y. Miyata, M. Nagatomo, T. Sakaguchi and M. Furukawa: Anal. Sci. 21 (2005) 907.

27 P. Rolfe, F. Scopesi and G. Serra: Meas. Sci. Technol. 18 (2007) 1683.

28 P. Rolfe: Med. Biol. Eng. Comput. 28 (1990) B34.

29 P. Rea, P. Rolfe and P. J. Goddard: Med. Biol. Eng. Comput. 23 (1985) 108.

30 P. G. Boswell, C. Szíjjartó, M. Jurisch, J. A. Gladysz, J. Rabai and P. Bühlmann. Anal. Chem. 80 (2008) 2084.

31 P. Rolfe: Biosensors in the Body: Continuous in vivo Monitoring, ed. D. M. Fraser (Wiley, Chichester, 1997) p. 217.

32 K. Rais-Bahrami, O. Rivera, G. T. Mikesell and B. L. Short: J. Perinatol. 22 (2002) 472.

33 P. Rolfe: Concise Encyclopedia of Medical and Dental Materials, ed. D. Williams (Pergamon Press, Oxford, 1990) p. 227.

34 P. Rolfe, M. Martin, D. Williams, R. Walters, K. Yamakoshi and S. Tanaka: In-vivo Chemical Sensors: Recent Developments, eds. S. J. Alcock and A. P. F. Turner (Cranfield Press, Bedford, 1993) p. 167.

35 R. Huch, W. Lübbers and A. Huch: Arch. Dis. Child. 49 (1974) 213.

36 R. Huch, A. Huch and P. Rolfe: Non-Invasive Physiological Measurements, ed. P. Rolfe (Academic Press, London, 1979) p. 313.

37 P. Eberhard: Anesth. Analg. 105 (2007) S48. 
38 V. Bernet, C. Döll, V. Cannizzaro, J. Ersch, B. Frey and M. Weiss: Pediatr. Anesth. 18 (2008) 872.

39 V. H. Tran, H. P. Chan, M. Thurston, P. Jackson, C. Lewis, D. Yates, G. Bell and P. S. Thomas: Sensors J. IEEE 10 (2010) 1514.

40 F. F. Jöbsis: Science 198 (1977) 1264.

41 P. Rolfe: Annu. Rev. Biomed. Eng. 2 (2000) 315.

42 T. Aoyagi: J. Anesth. 17 (2003) 259.

43 K. Yamakoshi and Y. Yamakoshi: J. Biomed. Opt. 11 (2006) 054028.

44 A. G. Tsai, P. C. Johnson and M. Intaglietta: Physiol. Rev. 83 (2003) 933.

45 A. J. van der Kleij, H. P. Kimmich, R. J. Goris, F. Kreuzer, J. de Koning and G. Beerthuizen: Adv. Exp. Med. Biol. 169 (1984) 869.

46 Y. Cui, Q. Wei, H. Park and C. M. Lieber: Science 293 (2001) 1289.

47 X. He, K. Wang, W. Tan, D. Xiao, J. Li and X. Yang: Chin. Sci. Bull. 46 (2001) 1962.

48 L. Mao, R. Yuan, Y. Chai, Y. Zhuo, X. Yang and S. Yuan: Talanta 80 (2010) 169.

49 F. Gao, L. Wang, L. Tang and C. Zhu: Microchim. Acta 152 (2005) 131.

50 R. V. Benjaminsen, H. Sun, J. R. Henriksen, N. M. Christensen, K. Almdal and T. L. Andresen: ACS Nano 5 (2011) 5864.

51 F. Patolsky, G. Zheng, O. Hayden, M. Lakadamyali, X. Zhuang and C. M. Lieber: Proc. Nat. Acad. Sci. 101 (2004) 14017.

52 B. J. Muller-Borer, M. C. Collins, P. R. Gunst, W. E. Cascio and A. P. Kypson: J. Nanobiotechnol. 5 (2007) 9.

53 R. Gill, M. Zayats and I. Willner: Angew. Chem. Int. Ed. 47 (2008) 7602.

54 C.-Y. Zhang, H.-C. Yeh, M. T. Kuroki and T.-H. Wang: Nat. Mater. 4 (2005) 826.

55 S. Sotiropoulou and N. A. Chaniotakis: Anal. Bioanal. Chem. 375 (2003) 103.

56 P. Rolfe: J. Meas. Sci. Technol. 17 (2006) 578.

57 P. Rolfe: Proc. SPIE 5630 (SPIE, Bellingham, WA, 2005) p. 439. 